\title{
Indications, Technique and Treatment Results of Argon Laser Trichiasis
} Siati Asmae ${ }^{1 *}$, Boukhani Insaf ${ }^{2}$, Habi Hind ${ }^{3}$, Jatik Badr $^{4}$, Mchachi Adil ${ }^{5}$, Benhmidoune Leila $^{6}$, Chakib Abderrahim ${ }^{7}$, Rachid Rayad ${ }^{8}$, El Belhadji Mohamed ${ }^{9}$

\author{
${ }_{1,2,3,4}$ Resident doctor, ${ }^{5,6,7,8,9}$ Associate professor, Department of Ophtalmology, Hospital August 20, UHC Ibn Rochd, Casablanca, \\ Morocco
}

DOI: $10.36347 /$ sasjs.2020.v06i03.002

| Received: 04.02.2020 | Accepted: 11.02.2020 | Published: 08.03.2020

*Corresponding author: Asmae Siati

Abstract

Original Research Article

Trichiasis or distichiasis is an abnormality characterized by an abnormal rubbing of the eyelashes against the eyeball, trichiasis is a generally acquired condition and the etiologies are multiple. In Morocco, trachoma remains the main cause of trichiasis, which can lead to corneal blindness. Among the therapeutic means, one finds the argon laser which offers several advantages: Ease of realization, reproducibility and limitation of recidivism. The objective of our work is to study the place of the Argon laser in the treatment of trichiasic and distichiasic eyelashes as an alternative to surgery, its indications, techniques and results.

Keywords: Trichiasis, distichiasis, argon laser.

Copyright @ 2020: This is an open-access article distributed under the terms of the Creative Commons Attribution license which permits unrestricted use, distribution, and reproduction in any medium for non-commercial use (NonCommercial, or CC-BY-NC) provided the original author and source are credited.

\section{INTRODUCTION}

The trichiasis is an acquired anomaly due to a change of direction of eyelashes originating from the ciliary margin and whose follicle sits in the anterior lamella and comes into contact with the ocular surface. It is a disorder of orientation of the follicle or aberration of the eyelash. It is often responsible for painful irritation of the corneoconjunctival surface. The trichiasis is a common complication of severe eyelid disorders: chemical burns, chronic inflammation, cicatricial pemphigoid and Stevens-Johnson syndrome. In Morocco, trachoma remains the main etiology of trichiasis, which can lead to corneal blindness. Several treatment modalities have been used to treat the trichiasic eyelashes. Each method has its own indications, success rate and complications. The destruction of argon laser ciliary bulbs offers many advantages and represents an adjuvant technique proposed in addition to or as an alternative to surgery when there are potential risks of aesthetic or functional damage.

The purpose of our work is to study this technique in the treatment of trichiasic eyelashes by specifying its advantages, indications and results.

\section{MATERIALS AND METHODS:}

This is a prospective study involving 180 patients (210 eyelids) over a period of study from
January 2011 to January 2019, who consulted for ciliary diseases for which argon laser treatment was indicated. Treatment consisted of argon laser destruction of the ciliary bulbs responsible for the disorders, involving the upper or lower eyelid or both.

Before starting treatment, the procedure and risks are explained to each patient. Careful interrogation and a complete ophthalmological examination allow, as far as possible, to determine the etiology of trichiasis. We used a topical anesthetic and a subcutaneous injection of $2 \%$ lidocaine in the trichiasis region to ensure strict patient immobility. Two types of lasers were used: blue green argon laser and multispots coupled to the slit lamp. We ask patients look from the opposite side of the eyelash to be treated and the eyelid is slightly turned outward to align the root of the trichiasic eyelashes. The spot parameters are: A high power of 0.5 to $1.2 \mathrm{Watt}$, the gap and exposure time is 0.2 seconds with a small spot diameter between 50 and 100 micrometer. The first spot is worn at the base of the eyelash to be treated and must be oblique with an angulation of 15 to $30^{\circ}$. It is enough to spray the eyelash and create a crater at the edge of the eyelid, then some impacts are applied deeper to create a crater 1.5 to $2.5 \mathrm{~mm}$ deep to ensure the destruction of the entire hair follicle, with an average of 10 to 30 spots per eyelash. The maximum number of eyelashes treated per eyelid state of 7 . The procedure is terminated by the application of antibiotic-corticoid ointment. This 
treatment is continued twice a day for one week. Each patient had monthly follow-up for 6 months and then every 2 months for 6 months. Recurrence has been defined as the regrowth of one or more trichiasic eyelashes after a maximum of two laser sessions with the same parameters.

\section{RESULTS}

A total of 210 eyelids of 180 patients were treated. $62 \%$ of the patients were men. The average age was 58 years old. The average duration of follow-up was 6 months. The etiological factor is found in 100 of our patients or $48 \%$, with chronic blepharitis (28 patients, $28 \%$ ), trachoma (20 patients, 20\%), chemical burns (8 patients, $8 \%$ ), and ophthalmic shingles (1 patient or $1 \%)$. In 43 patients $(43 \%)$ no cause was found (Figure-1).

All our patients had previously had at least one of the different treatment modalities (Figure-2). The result is considered good when there is no regrowth of the eyelash treated at 6 months of recoil and there is no associated palpebral complication. The treatment allowed the healing of 187 eyelids, 40 eyelids required a second session of argon laser treatment and the failure of 23 eyelids. The technique failure with regrowth of an already treated eyelash was observed in 18 eyelids that had healing after the third session. 5 eyelids had an indication failure with regrowth of additional eyelashes (4 eyelids required surgical treatment). In total, the success rate in our study is $93 \%$ after a maximum of 3 sessions without complications noted. The recurrence rate in our study was studied according to the etiologies and the different previous therapeutic modalities. It was elevated in patients who had a chemical burn (Figure3). It was also elevated in patients who had eyelid surgery (Figure-4).

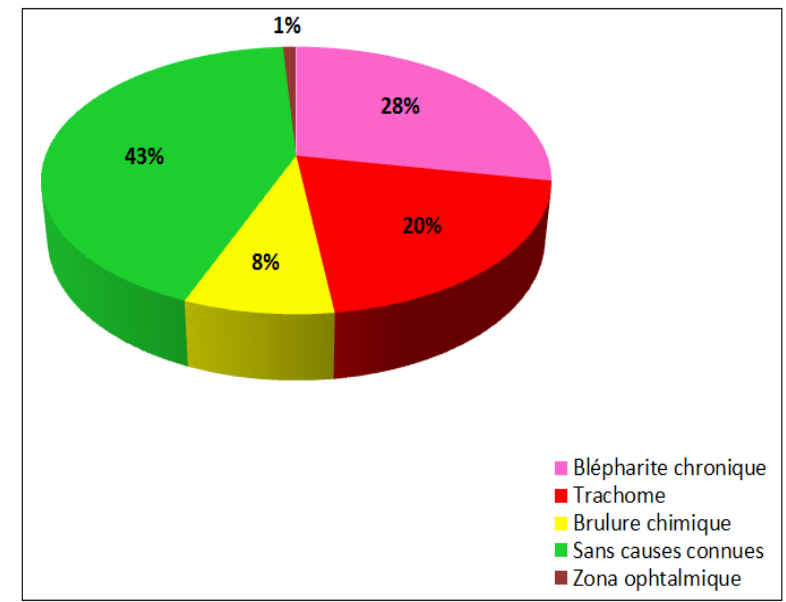

Fig-1: Etiological factors in our series
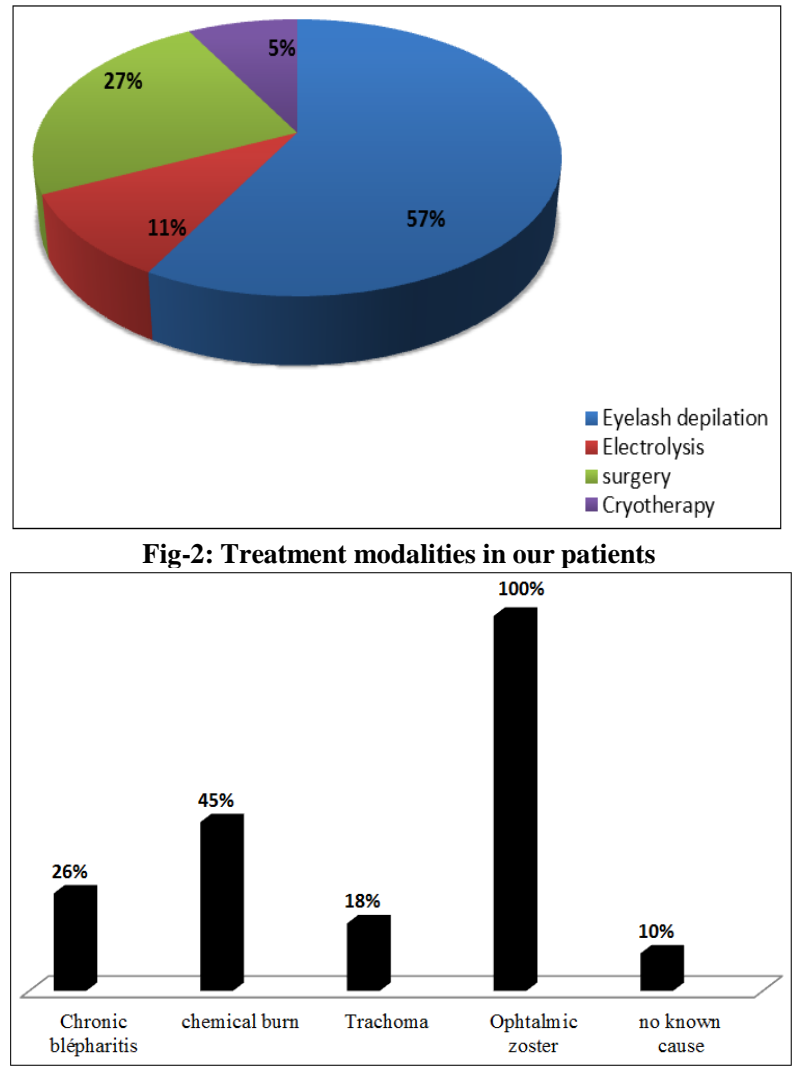

Fig-3: Recidivism rate according to etiology

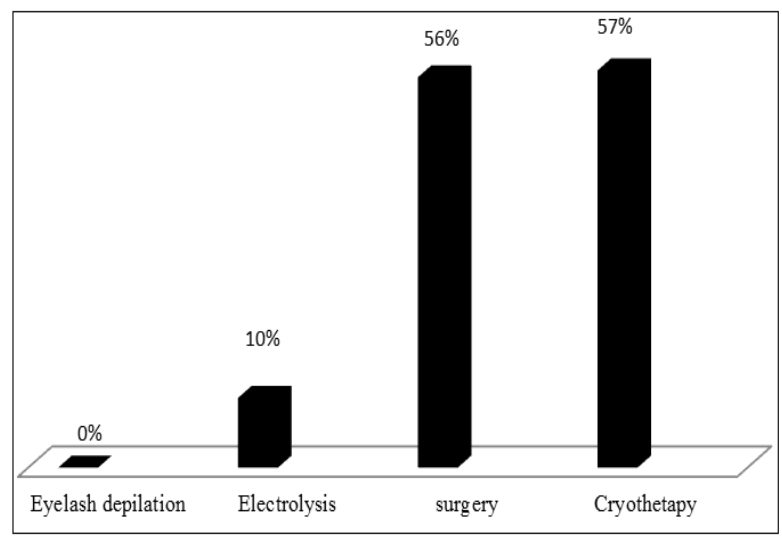

Fig-4: Recidivism rate according to previous therapeutic modalities

\section{DISCUSSION}

Characterized by an abnormal rubbing of the eyelashes against the eyeball, trichiasis is a generally acquired affection, whose etiologies are multiple [1]. In case of isolated (or pure) trichiasis, the palpebral free edge is in the normal position relative to the eyeball, unlike the entropion where there is a posterior tilt of the ciliary margin [2]. It should also be remembered that distichiasis is characterized by the existence of a second posterior row of abnormal lashes coming into contact with the eyeball. Topographically, trichiasis can be isolated (or pure), or on the contrary associated with palpebral disorders of static origin (entropion most often), or dynamic (palpebral retraction in particular, affecting preferentially the upper eyelid, especially in 
advanced trachoma). On the other hand, the isolated forms of trichiasis can be extended (or diffuse), that is to say affecting the three segments of the palpebral free edge; or on the contrary localized (or segmental), then affecting only one segment (nasal, central, or temporal) of the ciliary margin [1].

The treatment of trichiasis depends on many factors. In fact, it is necessary to take into account the type and extent of the latter, the presence of any reworkings sitting on the ciliary margin, the existence of retractions and / or associated palpebral mutilations, as well as possible conjunctival pathologies [3-6].

Indeed, as regards the potential etiologies of trichiasis, it is traditional to oppose the noninflammatory forms (the most frequent); whether they are of post-traumatic, post-operative, mechanical, or other origin; post-inflammatory andor post-cicatricial forms that may be observed in the context of trachoma or after certain chronic blepharitis, certain palpebroconjunctival burns, certain ocular pemphigoid, or even more rare pathologies, such as ulcerative colitis, Lyell syndrome or Stevens-Johnson syndrome [1]. In Morocco, trachoma remains the main cause of trichiasis.

Several treatment modalities have been used to treat the trichiasic eyelashes. Each method has its own indications, success rate and complications. Hair removal is a simple technique that offers temporary relief for 4 to 6 weeks. Electrolysis causes recurrence and excessive lysis of the palpebral tissues. Cryotherapy gives good results, but also complications especially on abnormal eyelids: Formation of scars [7], exaggeration of inflammation and destruction of the Meibomian glands. The surgical treatment offers good results, however it is limited in the particular areas (perimatic, canthus ...). Segmental tarsal rotation, for example, is contraindicated in the lower eyelid because, therapeutically, the management of segmental trichiasis (that is to say, extending over a distance of between 25 and $33 \%$ of the palpebral length) is based mainly on full-thickness pentagonal resection more or less associated with external cantholysis or minimally sliptranslation plasty [2, 8]. This intervention has the advantage of simplicity and is the guarantee of an acceptable esthetic result of the reconstructed eyelid in localized trichiasis minimal (that is to say, limited to $25 \%$ of the eyelid length) and in the localizations located at the level of the central segment of the eyelid (where the tarsus presents substantially the same height).

The Argon laser represents an adjuvant technique used in addition to or as an alternative to surgery, especially in the presence of potential risks of aesthetic or functional damage $[9,10,11]$. It is indicated at the lower and upper eyelid, increasing the power and duration of exposure to create a crater deeper
$2.5 \mathrm{~mm}$ to $3 \mathrm{~mm}$. The number of eyelashes must be less than or equal to 7 per eyelid. It is indicated as first-line therapy for chronic blepharitis, Stevens Johnson syndrome, chronic blepharitis, where surgical treatment may be responsible for significant eyelid damage. Surgery is reserved for recurrence or if entropion is associated $[9,10,12]$.

In our study, we investigated the thermoablation results of laser trichiasic eyelashes on a series of 180 patients over a 6-month follow-up period. The result is said to be satisfactory after 6 months of stability [9, 13-15]. The success rate in our work is $90 \%$ after two laser sessions and increases to $95 \%$ after the third session. This, joins the results of the literature. Campbell [13] reported a success rate of $80 \%$ with up to three sessions on 15 eyelids. Our success rate is lower than that reported by Gossman [16] who managed to reach a rate of $100 \%$ after two laser sessions, where he used higher parameters than usual: 100 micrometer in size, 0.5 seconds for the duration and 1.5 watts of power. However, $13 \%$ of patients had complications that were absent in our study. This is comparable to Yeung's study [17]. It has been reported that it is difficult to apply enough burns per eyelash to be effective. Ladas [18] said that the number of recurrences but also the success rate are closely correlated to the number of impacts needed to treat an eyelash.

The laser has several advantages. It is applied using the slit lamp with visibility that provides selective and precise destruction of the eyelash with good control without affecting healthy tissue. It is performed on an outpatient basis and does not always require infiltration anesthesia, done without suturing or dressing. However, this technique can't be used in bedridden or quine patients can not have a strict immobility.

\section{CONCLUSION}

The Argon laser is an effective technique used in addition to or as an alternative to surgery which allows a high precision with a good control of the treatment and a better cicatrization. It is a well-tolerated and easy to perform technique to avoid the high rate of complications secondary to other therapeutic modalities.

COPYRIGHT NOTICE: Authors who publish with this journal agree to the following terms.

\section{REFERENCES}

1. Adenis JP, Duprat F. Entropion et trichiasis. In: Encycl Méd Chir, Ophtalmologie. Ed: Elsevier, Paris, 1990:21 100 B20, 14.

2. Ruban JM, Misery L. Pathologie de l'appareil ciliaire. In: Encycl Méd Chir, Ophtalmologie. Ed: Elsevier, Paris, 1997:21 100 C20, 8.

3. Collin JRO. Entropion and trichiasis. In: A manual of systematic eyelid surgery. 2 nd edition. 
Churchill Livingstone, Edinburgh, London, Melbourne and New York, 1989:7-26.

4. Rodallec A, Krastinova D. La greffe chondromuqueuse. Son interêt dans la correction de l'entropion par cicatrice tarso-conjonctivale, du trichiasis et du distichiasis. J Fr Ophtalmol, 1983;6:87-93.

5. Goldberg RA, Joshi AR, McCann JD, Shorr N. Management of severe cicatricial entropion using shared mucocal grafts. Arch Ophthalmol, 1999;117: 1255-9.

6. West S, Taylor HR. Bilamellar tarsal rotation is the preferred treatment for trachomatous trichiasis. Surv Ophthalmol, 1999; 43:468-87.

7. Başar E, Özdemir H, Özkan Ş, Cicik E, Mirzataş Ç. Treatment of trichiasis with argon laser. European journal of ophthalmology. 2000;10(4):273-5.

8. Morax S, Benia L. Traumatisme et chirurgie plastique des paupières. In: Encycl Méd Chir, Ophtalmologie. Ed: Elsevier, Paris, 1985:21 100 D10, 11, 18p.

9. Charif Chefchaouni M, Belmekki M, Berraho A. Traitement des cils frottants du trichiasis par laser Argon. Journal de la SMO, 2006; 18, 92-95.

10. Ruban JM. Place du laser Argon en pathologie palpébrale. In: Pathologie orbito-palpébrale. Adénis JP, Morax S. Rapport Société Française d'Ophtalmologie, 1998. Masson Ed, Paris, 385-9.
11. Berry J. Recurrent trichiasis treatment with laser photocoagulation. Ophtalmic Surg, 1979;10:36-8

12. Ünlü K, Aksünger A, Söker S, Karaca C, Bilek K. Prospective evaluation of the argon laser treatment of trachomatous trichiasis. Japanese journal of ophthalmology. 2000 Nov 1;44(6):677-9.

13. Campbell DC. Thermoablation treatment for trichiasis using the Argon laser, Aust. NZ J. Ophtalmol, 1990; 18 (4): 427-430.

14. Sharif KW, Arafat AFA. The treatment of recurrent trichiasis with Argon laser photocoagulation. Eye, 1991;5:591-5.

15. Oshry T, Rosenthal G. Argon green laser photoepilation in the treatment of trachomatous trichiasis. Ophtalmol Plast Reconstr Surg, 1994;4:253-5.

16. Gossman WE, Hartmaier PJ, inventors; Openwave Tech Inc, assignee. System and method for providing data to a wireless device upon detection of activity of the device on a wireless network. United States patent US 6,317,594. 2001 Nov 13.

17. Yeung TT, inventor; Yeung, Teresa T., assignee. Partial to full thickness suture device \& method for endoscopic surgeries. United States patent US 5,895,395. 1999 Apr 20.

18. Ladas AK, Whipple B, Perry JD. The G spot: and other recent discoveries about human sexuality. Holt McDougal; 1982. 